\title{
FREE VIBRATIONS OF THE SLENDER NONPRISMATIC COLUMN UNDER THE PASSIVE AND ACTIVE FOLLOWER FORCE DIRECTED TOWARDS THE POSITIVE POLE
}

\author{
J. Szmidla*, A. Jurczyńska**
}

\begin{abstract}
The presented work deals with the issue of the free vibrations of slender nonprismatic column under active and passive load by the follower force directed towards the positive pole (the case of the specific load). The cross-section of the structure variable along the axis is realized by the division into prismatic segments of constant length and variable width described by the polynomial of degree I or II under the conditions of constant total length and volume. On the basis of the adopted physical model and the Bernoulli-Euler's theory, the mechanical energy of the considered column was determined. Taking into account Hamilton's principle (kinetic criterion of the stability loss), the differential equations of motion and natural boundary condition as well as the continuity conditions were obtained. The theoretical considerations and the results of the numerical simulations presented in this article allow to determine the influence of variable cross-section of the column and the geometry of structures implementing the external load on the values of natural frequencies and critical forces.
\end{abstract}

Keywords: free vibrations, slender systems, column, specific load, active and passive load

\section{Introduction}

Beams, rods and columns are widely used as structural elements in mechanical engineering, mining industry or civil engineering. Taking into account common applications of the slender systems, engineering practice requires special attention to the scientific problem of its free vibrations and stability. A lot of works refer to the searching for new design solutions, for example (Kasprzycki, 2011) where new designs of heads implementing various cases of the external loads were presented. In the work (Uzny et al., 2016) the influence of the method of defining the boundary problem on the values of the natural frequency was analyzed. An additional group of publications are works dedicated to the issue of the shape optimization and non-prismatic systems, e.g. (Szmidla, 2016; Mao, 2011; Sochacki, 2013) or (Ruocco et al., 2017) where the structures with variable cross-section have been studied. This work presents, for the first time, the results of numerical calculations regarding free vibrations of the non-prismatic system subjected to the active and passive specific load.

\section{Physical Model}

The slender nonprismatic column presented in Figure 1 is considered in this paper. In order to model cross-section variable along the axis, the system was divided into $n$ prismatic segments of constant length $l$ and thickness $h$ and variable width $b$ defined by the approximating function. Adopted division meets the conditions of constant total length and volume of the column. The shape approximation by the polynomial of degree I (linear function) and II (quadratic function) is considered.

Associate Prof. Janusz Szmidla, PhD. Eng.: Institute of Mechanics and Machinery Design Fundamentals, Częstochowa University of Technology, Dąbrowskiego73; 42-201 Częstochowa;PL,j.szmidla@imipkm.pcz.pl

** Anna Jurczyńska, MSc. Eng.: Institute of Mechanics and Machinery Design Fundamentals, Częstochowa University of Technology,Dąbrowskiego 73; 42-201, Częstochowa; PL, a.jurczynska@ imipkm.pcz.pl 


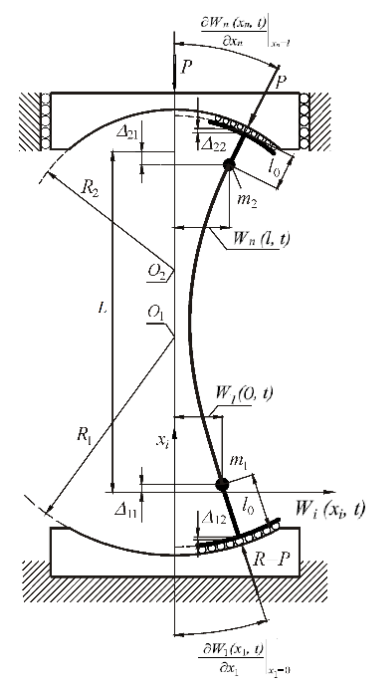

a)

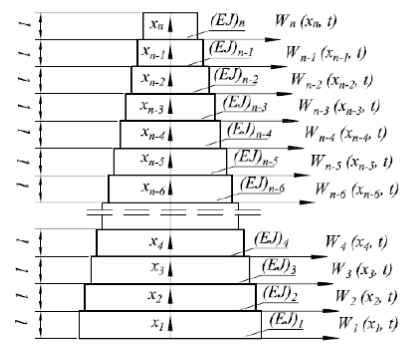

b)

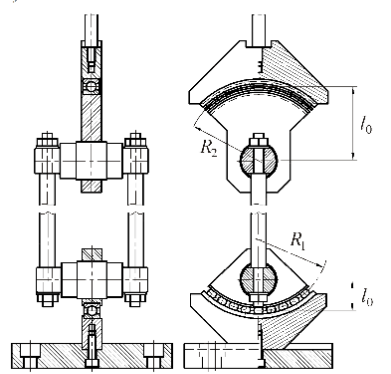

Fig. 1: Physical model of the column: a) division into segments b) loading structures.

The column is subjected to the follower force directed towards the positive pole (the case of the specific load defined by professor L. Tomski in 1996 (comp. (Bogacz et al., 1996)): active triggered by the load generating structure and passive implemented by the support structure (base reaction). Both systems responsible for generating the load are existing structures build of elements of circular outlines (slices of a rolling bearing). For the structural reasons the rigid elements of length $l_{0}$ enabling appropriate mounting of the column were also taken into account in the considerations.

\section{Problem Formulation}

On the basis of the physical model which description was given in chapter 2, the mechanical energy of the considered column was defined. The potential energy $V$ is a sum of energy of the bending elasticity of the particular segments of the system and the potential energy resulting from external loading:

$$
\begin{aligned}
& V=\frac{1}{2} \sum_{i=1}^{n}(E J)_{i} \int_{0}^{l}\left[\frac{\partial^{2} W_{i}\left(x_{i}, t\right)}{\partial x_{i}^{2}}\right]^{2} d x_{i}-\frac{P}{2}\left(\frac{1}{2} \sum_{i=1}^{n} \int_{0}^{l}\left[\frac{\partial W_{i}\left(x_{i}, t\right)}{\partial x_{i}}\right]^{2} d x_{i}\right)+ \\
& \left.+P \frac{l_{0}}{2}\left[\left(\left.\frac{\partial W_{1}\left(x_{1}, t\right)}{\partial x_{1}}\right|_{x_{1}=0}\right)^{2}+\left(\left.\frac{\partial W_{n}\left(x_{n}, t\right)}{\partial x_{n}}\right|^{x_{n}=l}\right)^{2}\right]+\frac{P}{2}\left[R_{1}\left(\left.\frac{\partial W_{1}\left(x_{1}, t\right)}{\partial x_{1}}\right|_{x_{1}=0}\right)^{2}+R_{2}\left(\frac{\partial W_{n}\left(x_{n}, t\right)}{\partial x_{n}}\right)^{x_{n}=l}\right)^{2}\right]
\end{aligned}
$$

The kinetic energy $T$ consists of the kinetic energy of all steps of the column as well as the kinetic energy of the concentrated mass $m_{1}, m_{2}$ that are modeling the weight of the structures implementing the load:

$$
T=\frac{1}{2} \sum_{i=1}^{n}(\rho A)_{i} \int_{0}^{l}\left[\frac{\partial W_{i}\left(x_{i}, t\right)}{\partial t}\right]^{2} d x_{i}+\frac{m_{1}}{2}\left[\frac{\partial W_{1}(0, t)}{\partial t}\right]^{2}+\frac{m_{2}}{2}\left[\frac{\partial W_{n}(l, t)}{\partial t}\right]^{2}
$$

The boundary problem was formulated taking into consideration the kinetic criterion of the stability loss (Hamilton's principle):

$$
\delta \int_{t_{1}}^{t_{2}}(T-V) d t=0
$$

By substituting equations $(1,2)$ into formula (3), after execution of the integration operation and the calculus of variation, the differential equations of motion of the particular segments were determined:

$$
(E J)_{i} \frac{\partial^{4} W_{i}\left(x_{i}, t\right)}{\partial x_{i}{ }^{4}}+\frac{P}{2} \frac{\partial^{2} W_{i}\left(x_{i}, t\right)}{\partial x_{i}^{2}}+(\rho A)_{i} \frac{\partial^{2} W_{i}\left(x_{i}, t\right)}{\partial t^{2}}=0, \quad i=1,2, \ldots, n
$$

The geometric boundary conditions (5) and the continuity conditions (6) referring to the deflection and the angles of deflection at the connection points of the particular steps were written in the following form: 


$$
\begin{gathered}
W_{1}(0, t)=-\left.\left(R_{1}-l_{0}\right) \frac{\partial W_{1}\left(x_{1}, t\right)}{\partial x_{1}}\right|_{x_{1}=0}, \quad W_{n}(l, t)=\left.\left(R_{2}-l_{0}\right) \frac{\partial W_{n}\left(x_{n}, t\right)}{\partial x_{n}}\right|^{x_{n}=l} \\
W_{j}(l, t)=W_{j+1}(0, t),\left.\quad \frac{\partial W_{j}\left(x_{j}, t\right)}{\partial x_{j}}\right|^{x_{j}=l}=\left.\frac{\partial W_{j+1}\left(x_{j+1}, t\right)}{\partial x_{j+1}}\right|_{x_{j+1}=0}, \quad j=1,2, \ldots, n-1
\end{gathered}
$$

Boundary conditions (5) result from geometric correspondences describing the construction of the loading and receiving heads. Having regard to the equations (5-6) in the formula (3), the remaining natural boundary conditions (7) and continuity conditions (8), necessary to solve the boundary value problem, were obtained:

$$
\begin{gathered}
\left.\left(R_{1}-l_{0}\right) \frac{\partial^{3} W_{1}\left(x_{1}, t\right)}{\partial x_{1}{ }^{3}}\right|_{x_{1}=0}+\left.\frac{\partial^{2} W_{1}\left(x_{1}, t\right)}{\partial x_{1}{ }^{2}}\right|_{x_{1}=0}+\left.m_{1}\left(R_{1}-l_{0}\right) \frac{1}{(E J)_{1}} \frac{\partial^{2} W_{1}\left(x_{1}, t\right)}{\partial t^{2}}\right|_{x_{1}=0}=0 \\
\left.\left(R_{2}-l_{0}\right) \frac{\partial^{3} W_{n}\left(x_{n}, t\right)}{\partial x_{n}{ }^{x_{n}}=l}\right|^{x_{n}=l}+\left.\frac{\partial^{2} W_{n}\left(x_{n}, t\right)}{\partial x_{n}{ }^{2}}\right|^{x_{n}=l}-\left.m_{2}\left(R_{2}-l_{0}\right) \frac{1}{(E J)_{n}} \frac{\partial^{2} W_{n}\left(x_{n}, t\right)}{\partial t^{2}}\right|^{x_{n}=l}=0 \\
\left.(E J)_{j} \frac{\partial^{2} W_{j}\left(x_{j}, t\right)}{\partial x_{j}{ }^{2}}\right|^{x_{j}=l}=\left.(E J)_{j+1} \frac{\partial^{2} W_{j+1}\left(x_{j+1}, t\right)}{\partial x_{j+1}^{2}}\right|_{x_{j+1}=0} \\
\left.(E J)_{j} \frac{\partial^{3} W_{j}\left(x_{j}, t\right)}{\partial x_{j}{ }^{3}}\right|^{x_{j}=l}=\left.(E J)_{j+1} \frac{\partial^{3} W_{j+1}\left(x_{j+1}, t\right)}{\partial x_{j+1}{ }^{3}}\right|_{x_{j+1}=0}, \quad j=1,2, \ldots, n-1
\end{gathered}
$$

Substituting the solutions of equations (4) into the boundary conditions $(5,7)$ and the continuity conditions $(6,8)$ allows one to obtain the system of equation. By comparing its characteristic determinant to zero, the transcendental equation for natural frequencies $\omega$ of the analyzed column.

\section{Results of Numerical Simulations}

In order to compare the obtained results, the following dimensionless parameters are introduced:

$$
\begin{gathered}
p^{*}=\frac{p}{L}, \quad q^{*}=\frac{q}{L}, \quad Z^{*}=\frac{b_{1}-b_{n}}{L} \cdot 100 \%, \quad \lambda=\frac{P \cdot L^{2}}{(E J)_{p r}}, \quad \Omega_{i}^{*}=\frac{(\rho A)_{p r} \omega_{i}{ }^{2} \cdot L^{4}}{(E J)_{p r}} \\
R_{i}^{*}=\frac{R_{i}-l_{0}}{L}, \quad m_{i}{ }^{*}=\frac{m_{i}}{L \cdot(\rho A)_{p r}}
\end{gathered}
$$

where the subscript ' $p r$ ' refers to the values defining properties of the prismatic comparative system of the same volume.
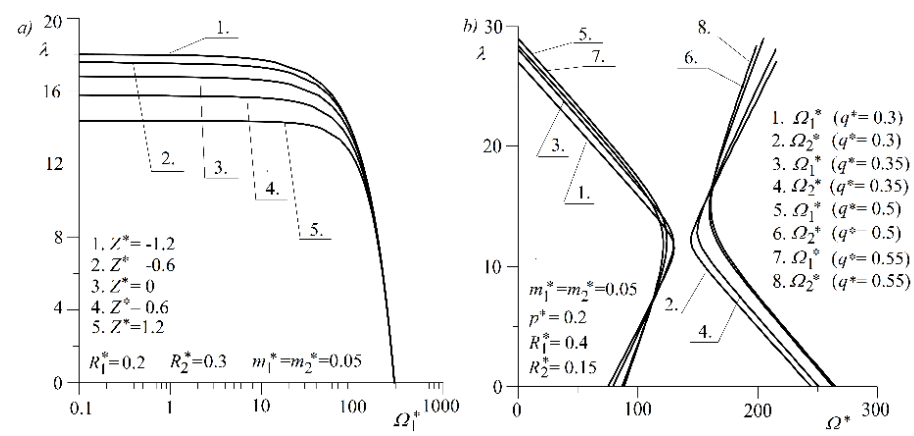

Fig. 2: The influence of variable cross-section of the system on the values of natural frequencies:

a) approximation by the linear function b) approximation by the quadratic function. 
In Figure 2 an influence of variable cross-section of the system approximated by the linear function (Fig. 2a) and polynomial of degree 2 (Fig. 2b) on the eigenvalues at given geometries of structures implementing active and passive load was presented. The graphs show that depending on the value of the coefficient defining the outline of the rod, the values of first two natural frequencies can increase or decrease in comparison to the prismatic comparative system. Therefore, it is possible to control the dynamic properties of the system by means of its appropriate shaping. The values of external load recorded at the natural frequency equal to zero refer to the critical loads. Based on the characteristic curves in the plane $\omega-\lambda$, the system can be classified as a divergent type or divergent pseudoflutter type.
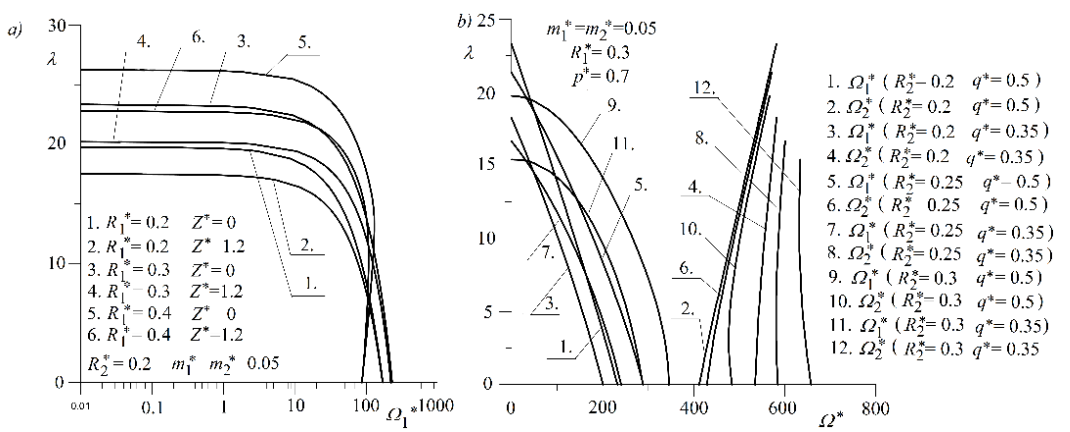

Fig. 3: The influence of variable geometry of the structures implementing external passive and active load on the values of first two natural frequencies.

Figure 3 presents the influence of the geometrical dimensions of the heads that are components of the loading (Fig. 3a) as well as the supporting structure (Fig. 3b) on the values of the natural frequencies of the non-prismatic column. Demonstrated data prove that the geometry of the structures implementing both active and passive load has the greatest impact on the dynamic properties of the system.

\section{Conclusions}

The aim of this work was the analysis of the slender nonprismatic subjected to the active and passive follower force directed towards the positive pole. Within the scope of the numerical computations it was shown that appropriate shaping of the outline of the rod can cause an increase or decrease in the value of the natural frequency in comparison with the reference column of the volume. An additional influence on the eigenvalues has the geometry of the structures implementing external load. Both factors make it possible to control the dynamic properties of the systems. On the basis of presented characteristic curves, the analyzed column was classified as a divergent type or divergent pseudoflutter type of systems.

\section{Acknowledgement}

The study has been carried out within statutory research BS/MN 1-101-302/17/P of the Institute of Mechanics and Machinery Design Fundamentals of Częstochowa University of Technology.

\section{References}

Bogacz R., Imiełowski B. and Tomski L. (1996) Stability and vibration of column structures subjected to generalized concentrated load, Proceedings of International Symposium Dynamics of Continua, Physikzentrum Bad Honnef, pp. 45-54

Kasprzycki A. and Tomski L. (2011) Chosen constructional solutions of the structures loading the Euler columns in vibrational and stability studies, Archives of Civil and Mechanical Engineering, XI (2), pp. 321-332.

Mao Q. (2011) Free vibration analysis of multiple-stepped beams by using Adomian decomposition method, Mathematical and Computer Modelling, vol. 54, pp. 756-764.

Ruocco E., Wang C. M., Zhang H. and Challamel N. (2017) An approximate model for optimizing Bernoulli columns against buckling, Engineering Structures, vol. 141, pp. 316-327.

Sochacki W. (2013) The dynamic stability of a stepped cantilever beam with attachment, Journal of Vibroengineering, vol. 15(1), pp. 280-290.

Szmidla J. and Jurczyńska A. (2016) Free vibrations of non-prismatic slender system subjected to the follower force directed towards the positive pole, Vibrations in Physical Systems, vol. XXVII, pp. 363-368.

Uzny S., Sokół K. and Osadnik M. (2016) Influence of amplitude on free vibration frequency of a partially tensioned column, Engineering Mechanics 2016, IT AS CR, Prague, pp.562-565. 